\title{
Visibility Analysis of the Oriental Pearl Based on Digital Landscape Simulation View from East Daming Road of Shanghai
}

\author{
LIU Song, ZHANG Shuwen \\ Department of Landscape Architecture, College of Urban Planning and Architecture , Tongji University ,No. 1239, Siping \\ Road ,200092,Shanghai, China -liusong5@tongji.edu.cn
}

KEY WORDS: visual analysis, City Image, ArcGIS, visibility, digital landscape, Scene Covere Index

\begin{abstract}
:
As the demand for visual quality of environment increases, visual analysis therefore plays progressively important role in current urban landscape construction and management. Guided by the City Image theory, this paper presents a covered scene index " $X$ " to describe the visibility of the target scene, and formulates a digital analysis model based on ArcGIS and 3D simulation. This method is applied to the viewpoint analysis from the East Daming Road of the North Bund to the Oriental Pearl in Shanghai and optimized solutions are proposed according to the results. It turns out that this simple and objective technique can serve as a good tool for the reference of urban landscape planning and management.
\end{abstract}

\section{INTRODUCTION}

As Spiro Kostof once said, City in any culture and any age always has its landmark, tall or towering, which highlight the city image (Spiro Kostof, 1999). The image of the city means the city in mind, which is formed by the perception of a city. Kevin Lynch put forward 5 elements of the image of the city, which is path, edge, domain, node, and landmark (Kevin Lynch, 1960). Landmarks are always tall buildings, which can arouse impressive images intuitively, such as Eiffel Tower in Paris, the Big Ben in London. Meanwhile, their height and special appearance make them useful in directing of sight and navigating in a city.

Shanghai is one of the most energetic cities in China, with the Bund and Lujiazui as its most famous area. The Oriental Pearl Tower and skyscrapers around it further brighten the city image. The west bank of Huangpu River is the most important route in the area, parallel to that is the East Daming Road,which is northwest of the North Bund, and is where the International Passenger Transport Center of Shanghai port resides. As visitors exits the distribution center, they hope to know their positions just by looking around. And the Oriental Pearl is hope to act as the landmark building to give passengers direction. But is it true in the existing condition? Here we set up a scene covered index to analyze visibility along the East Daming Road.

\section{RESEARCH STATUS}

Current research on visual analysis and control of urban environment mainly concentrate on the macroscopic and microscopic aspects. The macroscopic approach is represented by the height-control methods of western countries, including the strategic view from Britain, Fuseaux de Protection from France (Nishimura Yukio, 2005), and the visual analysis applied in exploiting Denny triangle in Seattle from the US (Xu Xiangbin, 2005). In the microscopic aspect, most researches combine the space with visual principles, such as the street dimension, degree of openness, etc. In 1979, Benedikt introduced the concept of Isovist, a way of measurement via the computation of the area and perimeter (Benedikt M L, 1979), which has become a common analytic technique in the design of urban squares and roads. On the basis of Isovist, Fisher established the horizon sphere, and defined the SOI (Spatial
Openness Index), to describe the openness of spaces (FisherGewirtzman D, 2003). Moreover, there are other concepts such as Teller's Sky Opening Index, and Zhang Xia's Visual Openness Index (Zhang Xia, 2010). In addition to the openness of spaces, the forward vision of human eyes is also a focus of research (Wong, 2005).

Among the analyses of visibility, applied the most is the visibility analysis of GIS. However, this method can only answer the question of "visible or not", rather than provide a description of the visual quality.

\section{VISIBILITY-THE PRINCIPLE OF SCENE COVERED INDEX}

The scenery covered analysis is also a point-to-point observation. But one of its features which stands out from the traditional visibility analysis is to depict visibility via a number of percentage, instead of a Boolean value. One can tell the degree of visibility from the number. The principle of this index can be shown by an elevation.

Target object can be seen or not basically depend on obstruction height (h) and it location (d) and the target object height $(\mathrm{H})$ and its distance from the viewpoint (D) (Fig.1). So we can get the covered scene index "X" (Zeng Shuhuai.2009).

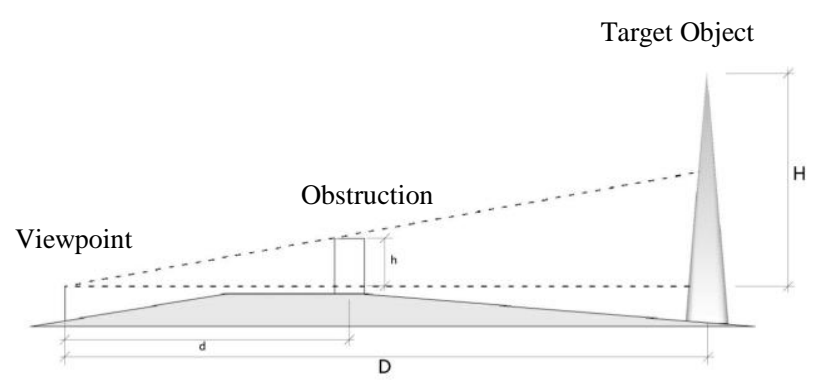

Figure 1. Calculating principles of covered scene analysis

The formula of covered relationship evaluation:

$$
\mathrm{h}=(\mathrm{H} \cdot \mathrm{d}) /(\mathrm{D} \cdot \mathrm{X})
$$


Where: $\mathrm{h}$ is the height of the obstruction relative to the viewpoint (here the height of viewpoint is $1.6 \mathrm{~m}$, equal to the mean height of people's eye), viewpoint,

$\mathrm{H}$ is the height of the target object relative to the

$\mathrm{d}$ is the horizontal distance between the obstruction and the viewpoint,

$\mathrm{D}$ is the horizontal distance between the target object and the viewpoint,

$$
\mathrm{X} \text { is the Covered Scene Index. }
$$

Thus we get:

$$
\mathrm{X}=(\mathrm{H} \cdot \mathrm{d}) /(\mathrm{D} \cdot \mathrm{h})
$$

When the locations of the viewpoint, the obstruction and the target object are fixed, we can calculate the value of the Covered Scene Index X according to their spatial relationship, thus the visual condition of the target object shows as following:

1) $0<\mathrm{X} \leq 1$ the target object is completely blocked. In this case the object does not appear in the vision, and cannot play a guiding role;

2) $1<\mathrm{X} \leq 2$ the target object's visual condition improves from invisible to upper-half-visible. In this case the object can barely be seen, indicating poor guiding ability;

3) $2 \leq X \leq 3$ the target object's visual condition improves from upper-half-visible to most-part-visible. In this case the object can be recognized easily, which has good guiding effect;

4) $\mathrm{X}>3$ the target object can almost be seen completely without any visual obstruction. The guiding ability becomes perfect. Together with surrounding landscapes, this forms a good viewing effect.

According to the theory of covered scene, we can analyze the visual degree of target scene; we can also control the height of buildings by fixing a certain value of covered index. Compared with Boolean values( 0 or 1 ) obtained in visibility analysis, the number we get here reflects the degree of visibility of the target object, and thus can provide more concrete visual information.

\section{METHODOLOGY: THE DIGITAL SIMULATION AND VISUAL ANALYSIS}

\subsection{Construction of the Digital Landscape Model of Research Area}

The digital landscape model is the basis of visual analysis. Due to their massive data storage, powerful ability of spatial analysis, and the fine control of models, we chose ArcGIS and Sketch Up as the tool to build up digital landscape models. These two softwares draw on each other's strength: they can not only represent the real scene, but also record, and process spatial data.

First the elevation data and orthophotography which covered the bund area are geo-processing adjusted, spatially positioned, and then superimposed in ArcGlobe to form the basic urban 3Denvironment of the research area. The positions of architectures are drawn in ArcMap according to the spatial positioned image, and then the architecture models are edited in Sketch Up. Then adding the surface texture to each building. At last import them back into ArcGlobe and then get the whole digital landscape model (Fig.2). Fig. 3 is the one sight of the digital landscape model.

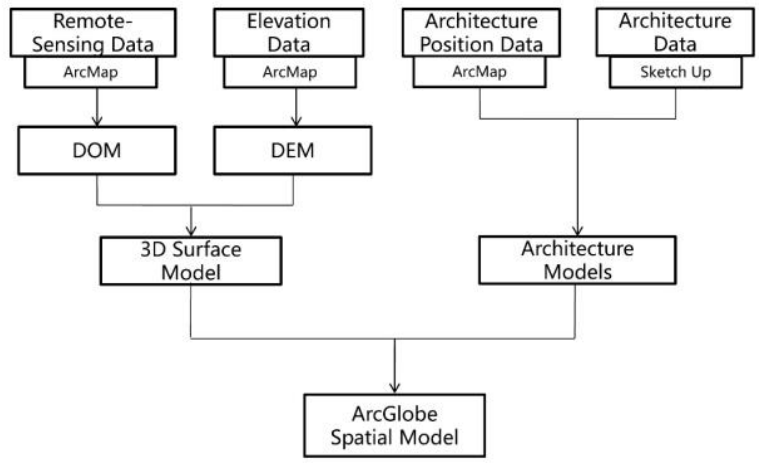

Figure 2. The process of building digital landscape model

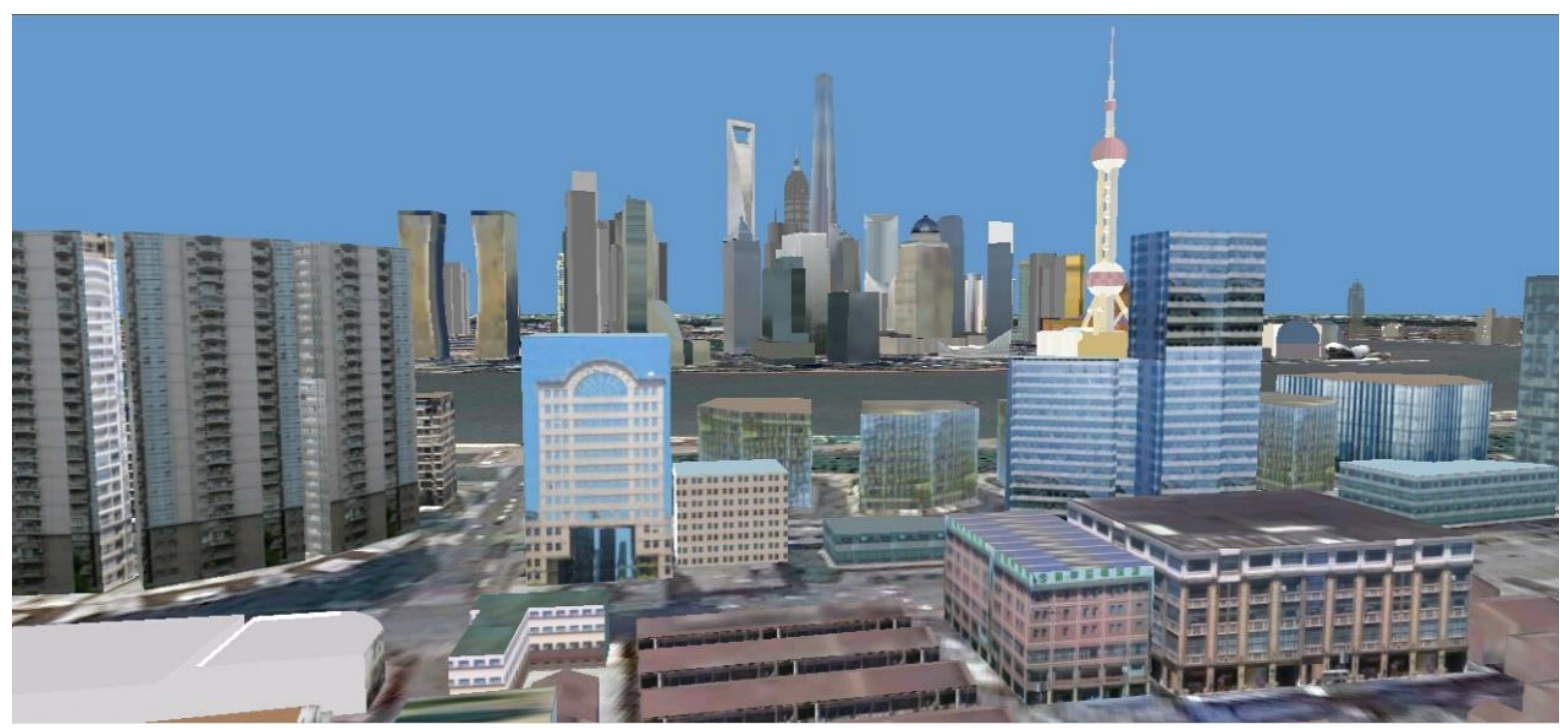

Figure 3. The process of building digital landscape model 


\subsection{Covered Scene index calculation}

Since there are no specific tools for covered scene analysis, we can establish visual analytic triangle, and take advantage of the powerful spatial analysis ability of ArcGIS, to compute the Covered Scene Index X from the point of basic geometry. Calculation follows as the following steps:

1) Building the digital landscape model of the bund area, recording the spatial data;

2) calculating the height $(\mathrm{h})$ of the obstruction relative to the viewpoint, the height $(\mathrm{H})$ of the target object relative to the viewpoint, the horizontal distance (d) between the obstruction and the viewpoint, the horizontal distance (D) between the target object and the viewpoint;

$3)$ Using the spatial data calculation function of the digital tools, and calculate $\mathrm{X}$ as $\mathrm{X}=((\mathrm{H}-1.6) \bullet \mathrm{d}) /((\mathrm{h}-1.6) \cdot \mathrm{D}))^{*}($ Fig. 4).

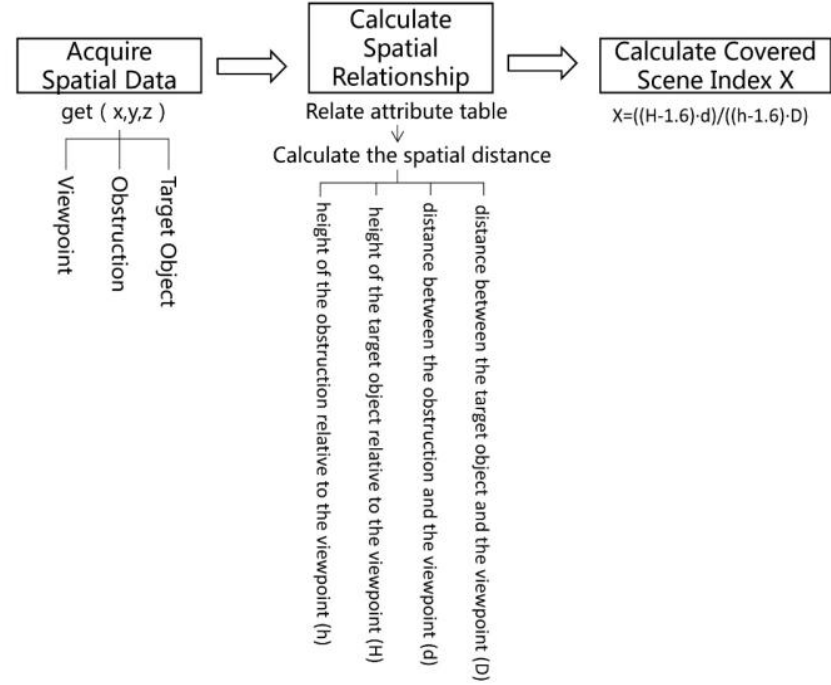

Fig.4 The process of covered scene index analysis

\section{CONCLUSSION}

\subsection{Landmark-Oriented Quantitative Visual Analysis of East Daming Road}

We took viewpoints every 60 meters along the East Daming Road. Totally we got 41 viewpoints (Fig 5). Covered Scene Indices of each viewpoint are mapped in Fig 6 which shows that the Oriental Pearl Tower is completely invisible from about 21 points' view.

Fig.6 also shows that separated by Xinjian Road, most points to the west of Xinjian Road have X greater than 1, some even greater than 2 , while points to the east of the road have $X$ less than 1 . That is to say, the visual quality on the west side is greater than the east where obstructed by high risings.

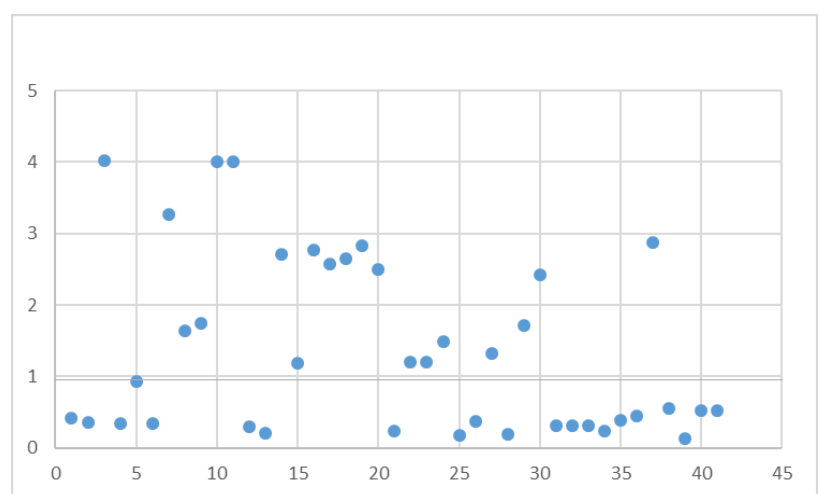

Fig.5 viewpoints and Classification of $\mathrm{X}$-values

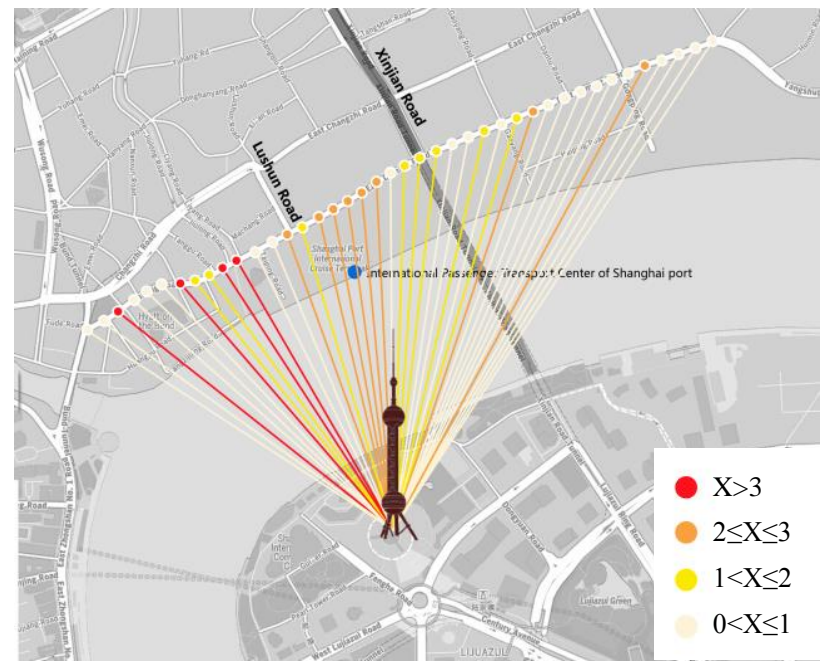

Fig.6 Distribution of X-values of 41 viewpoints

In the west of Xinjian Road, visitors can catch sight of 2/3 of the Oriental Pearl Tower at the intersection and the bridge, due to the low density of tall buildings and the small angle between north-south streets and sight lines towards the Oriental Pearl. There are several points $(2 \leq X \leq 3)$ in the section near the Shanghai Port, which reflects the broad vision in this part - one can even see the whole scene of skyscrapers in Lujiazui. So visitors can get their first overall impression of Lujiazui skyscrapers when they enter the East Daming Road from the Transport Center at Shanghai Port. As walking westwards, they can still have a sense of their location with the discontinuous visual guidance by the Oriental Pearl Tower (Fig.7). From the east of the Xinjian Road crossing, heavy tall buildings along the river further impact the visibility of the Oriental Pearl Tower. One can only glance at the steeple of the landmark, which cannot form a complete guidance of the city image.

\footnotetext{
${ }^{*} 1.6(\mathrm{~m})$ is the height of eyes.
} 

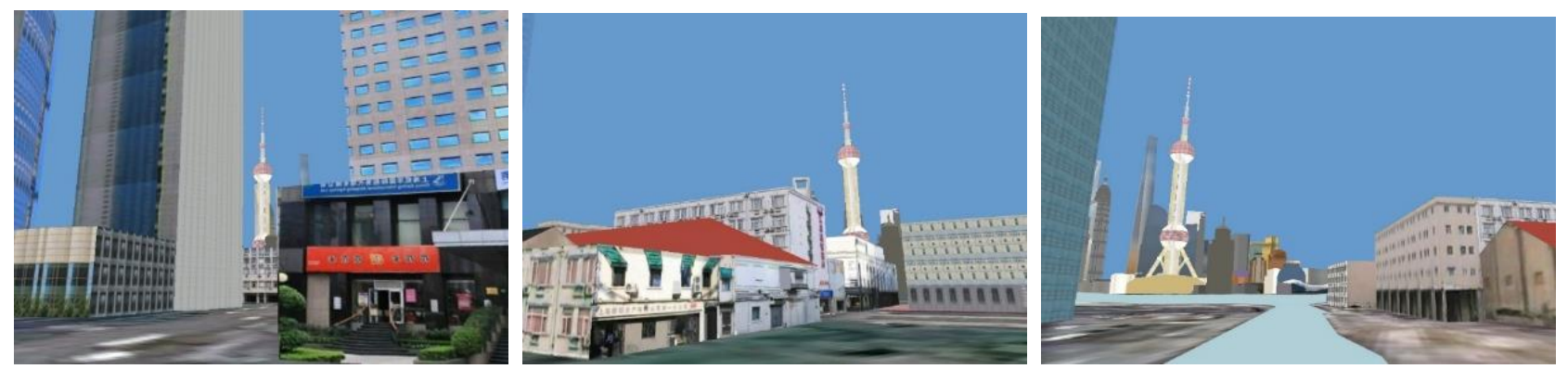

Fig.7 visibility of the landmark from the west of Xinjian Road

\subsection{Suggestions for Visual Guidance}

According to the analysis above, we put forward the following suggestions for the street reconstruction, aimed to strengthen the visual guidance of the Oriental Pearl Tower to visitors along East Daming Road.

\subsubsection{More Open Space and More High-Quality Viewpoints}

Because of a green space near the riverside, the vision is quite satisfactory near the International Passenger Transport Center of Shanghai Port. So keep the green space and design a pocket garden or squares and set up stopover points at the block under construction on the north side of the road (Fig.8). Meanwhile we can attract visitors' sight to the Oriental Pearl Tower and Lujiazui skyscrapers by square arrangements and plantings, etc. Moreover, the open space here can be used as a distribution center of the Transport Center of Shanghai Port.

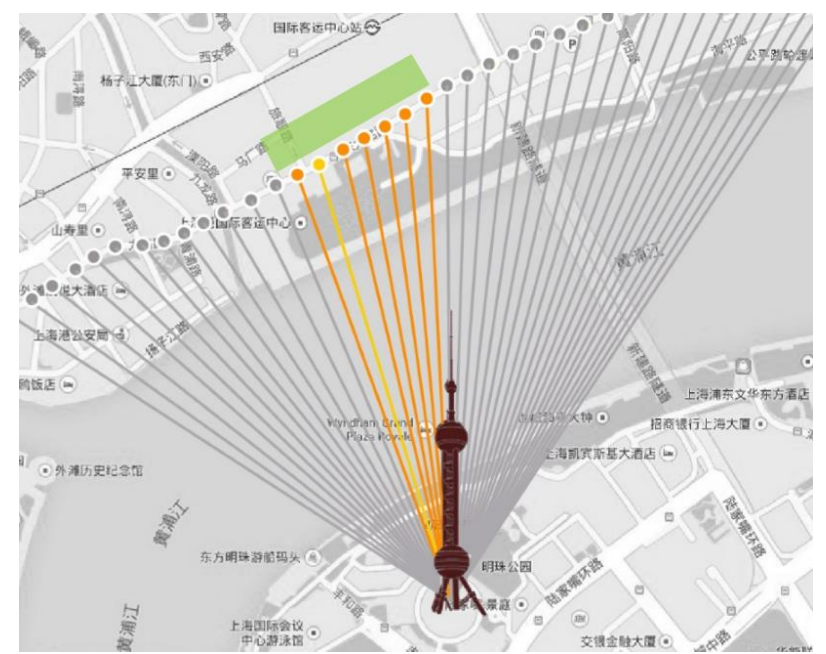

Figure 8. Suggested locations of green space and square
Points to the west of Xinjian Road, which have high X-values, are mostly near intersections and bridges. They can be constructed as open spaces, and become high-quality viewpoints, if one appropriately deals with the tempo of pausing and passing, and the relationship between pedestrians and vehicles. In this way the viewing comfort would be greatly improved.

\subsubsection{Sparse Buildings and Vision Rhythm}

Buildings to the east of Xinjian Road have become a screen between the landmark and East Daming Road, severely blocking visitors' sights. The director function of the landmark has been totally lost along this road.

The suggestion is to reduce the density of tall buildings, and increase the frequency of points $(2 \leq \mathrm{X} \leq 3)$, leading to the case where points $(2 \leq X \leq 3)$, points $(1 \leq X \leq 2)$ and points $(0 \leq X \leq 1)$ appear alternatively. The height of buildings can be computed and controlled in terms of these fixed values of covered scene indices. From the perspective of form aesthetics, repeated and continuous appearance of the target object and occasional vision guidance can give rise to a sense of rhythm, and increase the degree of beauty and reorganization of this area.

On the other hand, the Gestalt law tells us that the integrity of experiences and behaviours is the character of perception, and what you have perceived is more than what you have seen. Humans' psychological activities have their entire process inside, which is the precondition of experience. Therefore, when people walk along the road and glance at the skyscrapers of Lujiazui at a certain frequency (fig.9), they will subconsciously unite them into a whole by perception, and get an overall image of the skyscrapers.

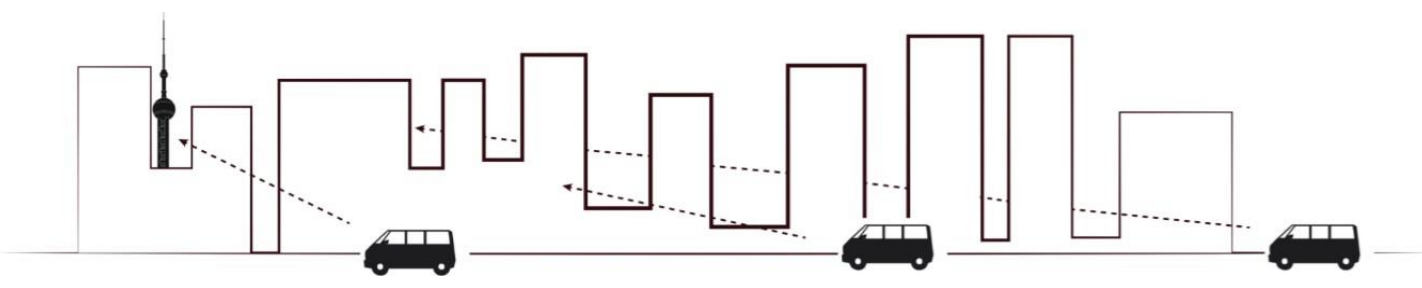

Fig.9 Repeated and continuous visual guidance 


\section{DISCUSSION}

This research explores the technique of digital landscape simulation and the realization of the quantitative analysis of the degree of visibility. We put forward an index of covered scene to measure the degree of visibility according to the basic visual rules in ArcGIS. Through the application of digital techniques, we can find out the visual condition of the Oriental Pearl along the East Daming Road by this simple and objective method, and provide a reference for the design and reconstruction of the street and open spaces.

The accuracy of the simulation is an important premise in the digital analysis. This research adopt the geographic coordinate system of WGS1984 to ensure the accuracy of the location. However, we are unable to modelled plants and vegetation into the system due to the restriction of the computer competence and the difficulty of modelling a living organisms. Nevertheless, compared with the traditional method of site visiting, digital analysis is fast, objective and convenient. It can be used in urban visual analysis, especially in the protection of existing visual environment in city achievements, and the comparison and selection of different alternatives.

\section{REFERENCES}

Kostof, S., 1999. The City Shaped: Urban Patterns and Meanings Through History. Thames \& Hudson, New York, NY, USA.

Kevin Lynch, 1960. The Image of the City. The MIT Press, Cambridge, MA, USA.

Nishimura Yukio, 2005. Conservation Planning for Urban Landscape in the Western Countries. Shanghai science and Technology Press, Shanghai, China.

Xu Xiangbin, Dai Zhizhong, 2005. The using of scenery density analysis in mountainous city sight control. Chongqing Architecture, 2005(04), pp. 21-28.

Benedikt M L, 1979. To take hold of space: isovists and isovist fields. Environment and Planning B. 1979, 6(1), pp. 47-65.

Fisher-Gewirtzman D, 2003. Wagner I A. Spatial openness as a practical metric for evaluating built-up environments. Environment and Planning B: Planning \& Design. 2003(1), pp. 37-49.

Zhang Xia, Zhu Qing, 2010. Visual analysis for urban environment based on the 3DGIS. International Urban Planning. 2010, 25(1), pp. 66-70.

Wong , 2005. The visual quality of urban park scenes of Kowloon Park, Hong Kong: likeability, affective appraisal, and cross-cultural perspectives. Environment and Planning B: Planning and Design. 2005(32), pp. 617-632.

Zeng Shuhuai, 2009. Research on methods and application of analysis and control by sight in urban design. South Architecture, 2009(01), pp. 17-20. 